\title{
High incorporation of dietary 1-O-heptadecyl glycerol into tissue plasmalogens of young rats
}

\author{
Arun K. Das and Amiya K. Hajra \\ Neuroscience Laboratory, Mental Health Research Institute and Department of Biological Chemistry, \\ University of Michigan, Ann Arbor, MI 48109, USA
}

Received 30 November 1987

\begin{abstract}
When 1-O-heptadecyl-rac-glycerol was fed $(20 \mathrm{mg} / \mathrm{g}$ of food) to 19-day-old rats for 10 days, a high incorporation of the heptadecyl group into the 1-O-alk-1'-enyl group of ethanolamine plasmalogens of all tissues was observed. For example, $62 \%$ of the alkenyl groups from liver plasmalogen was of the 17:0 variety. The analogous values for other tissues were $62 \%$ in kidney, $57 \%$ in lung, $57 \%$ in heart, $50 \%$ in intestine, $43 \%$ in erythrocytes, $25 \%$ in testis and $8 \%$ in brain. The corresponding figures in the control rats (fed normal rat chow) were only 2-3\% of 17:0 for all tissues. Available evidence indicates that dietary 1-O-heptadecyl-sn-glycerol is utilized to form tissue plasmalogens without the cleavage of the ether bond. The relevance of these results to the possible dietary ether lipid therapy of patients suffering from congenital ether lipid deficiency is discussed.
\end{abstract}

Dietary ether lipid; Heptadecylglycerol; Ether lipid incorporation; Plasmalogen; (Rat tissue)

\section{INTRODUCTION}

It has been long known from the work of Blomstrand and co-workers that dietary glycerol ether lipids are absorbed intact from the digestive tract of humans and rats without the cleavage of the ether bond [1,2]. Later, Paltauf [3] and Mangold and co-workers [4,5] have shown that in intestinal mucosal cells, dietary 1-O-alkyl-snglycerol is metabolized to plasmalogens (1-Oalk-1'-enyl-sn-glycerol derivatives), the major membrane ether lipid. However, little is known regarding the transport and conversion of dietary ether lipids to membrane ether lipids in different tissues other than intestine. There is a brief report

Correspondence address: A.K. Hajra, Neuroscience Laboratory, Mental Health Research Institute and Department of Biological Chemistry, University of Michigan, Ann Arbor, MI 48109, USA

Abbreviations: TLC, thin-layer chromatography; GLC, gasliquid chromatography; $\mathrm{PE}$, phosphatidyl ethanolamine by Bandi and co-workers [6] who showed that, in rats, a small fraction of the dietary ether lipid is incorporated into plasmalogens of liver. On the other hand, using tracer doses of radioactive ether lipids, a number of workers have concluded that in animals, exogenous ether lipids are not incorporated into tissue ether lipids to any significant extent $[7,8]$.

In recent years, the possible incorporation of dietary ether lipids to membrane glycerol-ether lipids and plasmalogens has generated much interest because of the discovery of a number of genetic diseases such as Zellweger cerebrohepatorenal syndrome where the enzymes catalyzing the ether bond synthesis are deficient $[9,10]$ and hencc the ether lipids are found to be practically absent in the tissues of these patients [11]. A preliminary study indicated that by supplementing the diet of these patients with batyl alcohol (1-O-octadecyl$s n$-glycerol), the erythrocytes' plasmalogen content can be restored to almost normal levels [12]. However, nothing is known about the changes in the ether lipids in other tissues of these patients. 
We, therefore, started experiments with young rats to investigate the incorporation of dietary $O$-alkyl glycerol into plasmalogens of different tissues. For this purpose, we chose 1-O-heptadecyl glycerol, since the heptadecyl moiety is almost absent in naturally occurring ether lipids but the chain length resembles the chain length distribution of membrane ether lipids which is mainly 16:0, 18:0 and 18:1. The results from these experiments, as reported here, show that not only is dietary heptadecyl glycerol incorporated into plasmalogens of all tissues but the extent of incorporation is remarkably high.

\section{MATERIALS AND METHODS}

$n$-Heptadecanol, methanesulfonyl chloride and rac-isopropylidene glycerol were obtained from Aldrich (Milwaukee, WI). 2,3-Isopropylidene-sn-glycerol, 1,2-isopropylidene-sn-glycerol and lysophosphatidyl ethanolamine were purchased from Sigma (St. Louis, MO). All solvents were analytical grade and freshly distilled before use. Other materials were the same as described [13].

1-O-Heptadecyl-rac-glycerol and its pure optical isomers (1-sn and 3-sn glycerols) are prepared by the condensation of heptadecyl methanesulfonate with the corresponding isopropylidene glycerols followed by acid hydrolysis as described by Baumann and Mangold [14]. The purity of the final compounds was at least $98 \%$ as determined by TLC and GLC.

Sprague-Dawley 19-day-old male rats (just after weaning), weighing 30-35 g, obtained from Harlan Sprague-Dawley (Indianapolis, IN) were used for these studies. The animals were divided into groups of 4 rats. The control group was given powdered chow and water and the experimental group received the same diet but containing 1-O-heptadecyl-(rac or 1-sn or 3-sn)-glycerol and water. All the groups were fed ad libitum for $5-10$ days as indicated in fig. 1 and table 1 . The animals consumed about $4 \mathrm{~g}$ food/day per rat at the beginning, which was increased to about $9 \mathrm{~g} /$ day per rat at the end (10 days) of the experiment. The average weight of the animals at the end (10 days) of the experiment was $85 \mathrm{~g}$.

At the end of the feeding period, the animals were lightly anesthetized with ether and killed by decapitation. The blood and other tissues (liver, kidney, brain, testis, heart, lung and intestine) were collected immediately and, if necessary, were stored at $-70^{\circ} \mathrm{C}$. The total lipid was extracted from the pooled tissues of the individual classes with neutral chloroformmethanol $(1: 1)$ and washed as described [13].

The chain length of the alkenyl group of PE plasmalogen was analyzed by first subjecting the total lipid extract to alkaline methanolysis [15], isolating the resulting lysoPE plasmalogen by TLC [13], followed by acid hydrolysis [16] and the reduction of the liberated aldehydes by $\mathrm{NaBH}_{4}$ to the corresponding alcohols [13]. The alcohols, after converting to acetate esters [17], were separated according to the chain lengths by GLC. The GLC analyses were carried out on a Hewlett-Packard gas chromatograph, model $5710 \mathrm{~A}$, equipped with a flame ioniza- tion detector and an electronic integrator (Spectra Physics). The alcohol acetates were analyzed on $15 \%$ Silar $10 \mathrm{C}$ supported on Gas Chrom $\mathbf{R}$ in a glass-lined stainless steel analytical column $(6 \mathrm{ft} \times 0.125 \mathrm{in})$ with temperature programming. The peaks were identified by comparing the retention values to that of known standards.

The plasmalogen content of total lipid extracts was determined by the two-dimensional TLC method as described by Horrocks [18]. The lipid phosphates were measured as described by Bartlett [19]. Other methods were the same as described $[13,20]$.

\section{RESULTS AND DISCUSSION}

The chain length distribution patterns of the 1-O-alk-1'-enyl group of the ethanolamine plasmalogen from different tissues of the control and experimental (1-O-heptadecyl-rac-glycerolfed) rats are shown in fig. 1 . Only the major species are shown here which comprise $>90 \%$ of the total alkenyl groups of PE plasmalogen. As reported by many authors [21], and as is the case here, the chain length distribution of the alkenyl group in control rats constitutes mainly three isomers, i.e. $16: 0,18: 0$ and 18:1 (fig.1). Only in intestine is 20:0 present as an additional component (fig.1), which has been reported by Paltauf [3]. In contrast, the alkenyl group of PE plasmalogen from most of the tissues of rats which were chronically fed 1-O-heptadecyl glycerol contained 17:0 as the major species (fig.1). The lower incorporation in the testis and brain is probably because testis is immature in these young rats, and the formation of brain myelin, the major source of brain plasmalogens, is almost complete before the start of the experiment (19-day-old rats). Administration of alkyl glycerol to rats before the beginning of myelination ( $<10$-day-old rats) would probably result in a large incorporation of the exogenous ether lipid to brain lipids. However, it is difficult to feed chronic doses of ether lipids to these suckling rats.

Though a high amount of alkyl glycerol is fed to these rats, the amount of plasmalogens per $g$ of tissue, measured according to the method of Horrocks [18], was found to be the same in the control and experimental animals. The incorporation of the dietary ether lipid into tissue plasmalogen is very rapid, as we observed in a separate experiment that feeding the same amount of heptadecyl glycerol per day to these young rats for only 5 days increased the 17:0 content of the alk-1' -enyl group 

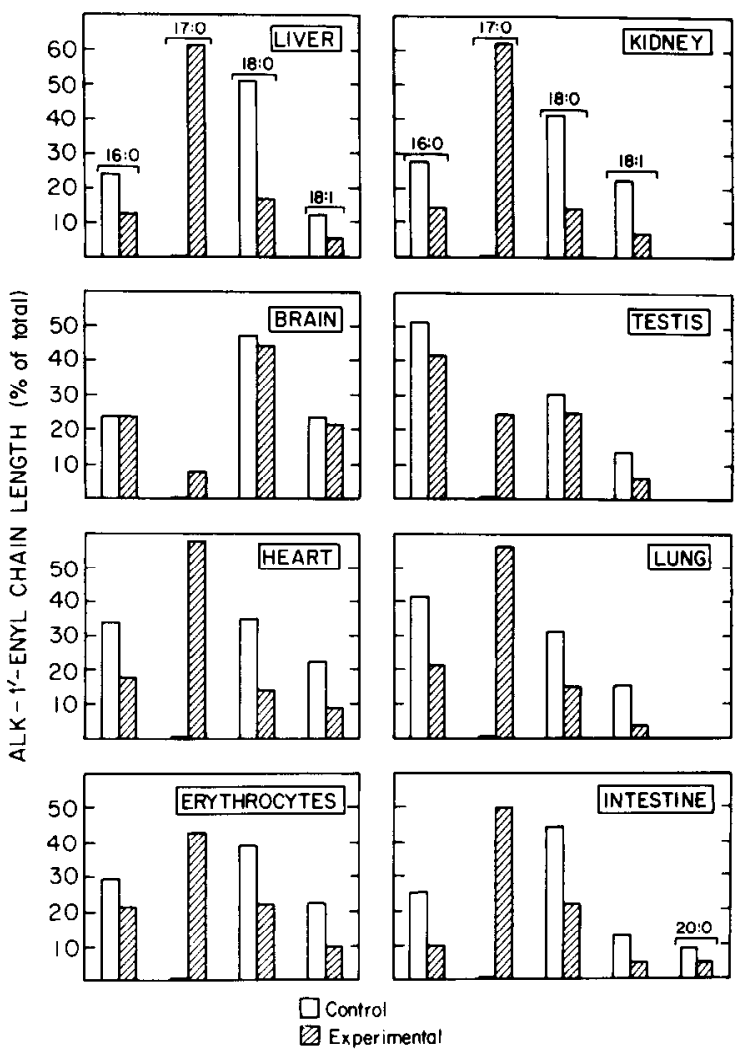

Fig.1. The chain length distribution of the alk-1'-enyl group at the $\mathrm{C}-1$ position of PE plasmalogen from different tissues of rats fed either powdered food (open bars) or powdered food containing $2 \%(\mathrm{w} / \mathrm{w})$ 1-O-heptadecyl-rac-glycerol (hatched bars) for 10 days. The chain lengths are indicated at the top panel using the usual nomenclature, i.e. number of carbon atoms: number of double bonds other than at the $l^{\prime}$-position. of kidney and liver lipid to $50-55 \%$. We also found that when fed $1 \%$ with the diet for 5 days, the natural isomer of the heptadecyl glycerol, i.e. 1-O-heptadecyl-sn-glycerol is incorporated to considerable extent into tissue plasmalogens $(-55 \%$ in liver and kidney) but the unnatural isomer, 3-Oheptadecyl-sn-glycerol, is incorporated only in low amount $(\sim 7 \%$ over the controls) in these tissues (table 1). A similar high (40-50\%) incorporation of the natural 1-alkyl-sn isomer (but not the 3-sn isomer) into tissue plasmalogens of other tissues (heart, lung, spleen, skeletal muscle, intestine, erythrocytes, etc.) was also observed (not shown). As found for the DL-mixture (fig.1), the incorporation of the 1-sn isomer into brain lipid is also low but significantly higher than that from the 3-sn isomer (table 1). These findings are similar to the results reported by Paltauf [3], who showed that in intestinal mucosal cells only the 1-sn isomer of the 1-O-octadecyl glycerol, but not the 3-sn isomer, was incorporated into plasmalogens without the cleavage of the ether bond. Apparently, 1-O-alkyl$s n$-glycerol is enzymatically converted to $1-O$ alkyl-sn-glycerol-3-phosphate [22], which is further stereospecifically acylated in tissues to 1-Oalkyl-2-acyl-sn-glycerol-3-phosphate [23], the key precursor of glycerol ether lipids and plasmalogens [24]. However, the enzyme which catalyzes the oxidative cleavage of ether bond of alkyl glycerol is not stereospecific [25], so that the administered 3-sn isomer of the alkyl glycerol is completely cleaved in the body to form heptadecanoic acid and glycerol. Our finding of a much higher incorporation of the 1-sn isomer compared to the 3-sn

Table 1

Incorporation of dietary 1-O- and 3-O-heptadecyl-sn-glycerol into PE plasmalogen of rat tissues

\begin{tabular}{|c|c|c|c|c|c|c|c|c|c|c|}
\hline \multirow[t]{3}{*}{ Tissues } & \multicolumn{10}{|c|}{ Carbon number of alk-1 'enyl group ( $\%$ of total) } \\
\hline & \multicolumn{2}{|c|}{$16: 0$} & \multicolumn{2}{|c|}{$17: 0$} & \multicolumn{2}{|c|}{$18: 0$} & \multicolumn{2}{|c|}{$18: 1$} & \multicolumn{2}{|c|}{$20: 0$} \\
\hline & $1-s n$ & $3-s n$ & $1-s n$ & $3-s n$ & $1-s n$ & $3-s n$ & $1-s n$ & $3-s n$ & $1-s n$ & $3-s n$ \\
\hline Liver & 15.8 & 26.3 & 55.3 & 7.3 & 16.9 & 38.3 & 3.8 & 17.0 & 0.5 & 1.2 \\
\hline Kidney & 17.3 & 32.0 & 55.9 & 7.1 & 15.9 & 31.2 & 7.6 & 17.7 & 0.3 & 2.2 \\
\hline Brain & 28.3 & 29.1 & 5.5 & 0.9 & 38.0 & 41.8 & 20.0 & 19.7 & 0.5 & 0.4 \\
\hline
\end{tabular}

Rats were fed (10 mg per $\mathrm{g}$ of powdered food) either 1-O-heptadecyl-sn-glycerol (1-sn) or 3-Oheptadecyl-sn-glycerol (3-sn) or powdered food only (control group, not shown) for 5 days and then the alk-1'-enyl group compositions at the C-1 of PE plasmalogen from different tissues were analyzed as described in the text 
isomer indicates that only the dietary 1-Oheptadecyl-sn-glycerol is incorporated into the membrane ether lipids with the ether bond remaining intact during such conversions.

The results presented above show that in young animals dietary ether lipids, when fed in a chronic dose, are utilized by all tissues to synthesize membrane ether phosphoglycerides. The choice of heptadecyl glycerol is fortuitous because the chain length of the $O$-alkyl group seems appropriate not only for the incorporation into the alkyl glycerol ethers but also to their dehydrogenation products, i.e. plasmalogens. This is in contrast to the findings of Weber [26] who showed that relatively shorter chain alkyl glycerol (1-O-dodecyl glycerol), when fed to the rats, is incorporated to the alkyl glycerol ethers but not to the plasmalogens of different tissues. It seems that the very narrow and specific chain length distribution of the alkenyl group of plasmalogens is not only due to the specificity of the acyl CoA reductase [27], but also due to the specificity of the dehydrogenase which catalyzes the formation of the alk-1'-enyl group from the corresponding ethanolamine glycerol ethers [28]. Reports by Mangold and co-workers $[4,6,29]$ also indicate that the dehydrogenase is very specific with respect to the chain length and the degree of unsaturation of the alkyl group present in the ether lipid.

These findings as reported here are somewhat surprising because an unnatural dietary glycerol ether is found to be incorporated into tissues to such a high extent that it became the major component of membrane plasmalogens. This finding will be useful to study the physiological effects of changing the even numbered chain length of a membrane lipid to an odd numbered one and also to study the in vivo turnover of plasmalogens in tissues as the unnatural 17:0 moiety probably would be recycled at a very low rate. The present finding has also clinical relevance to a possible dietary ether lipid therapy for a number of genetic diseases such as Zellweger cerebrohepatorenal syndrome, neonatal adrenoleukodystrophy, Refsum disease and Rhizomelic chondrodysplasia punctata, where there is a deficiency of ether lipids in all tissues $[10,30]$.

Acknowledgements: This work is supported by NIH grants NS 08841 and NS 15747.

\section{REFERENCES}

[1] Bergstrom, S. and Blomstrand, R. (1956) Acta Physiol. Scand. 38, 166-172.

[2] Blomstrand, R. and Ahrens, E.H. jr (1959) Proc. Soc. Exp. Biol. Med. 100, 802-805.

[3] Paltauf, F. (1971) Biochim. Biophys. Acta 239, 38-46.

[4] Bandi, Z.L., Aaes-Jorgensen, E. and Mangold, H.K. (1971) Biochim. Biophys. Acta 239, 357-367.

[5] Mangold, H.K. (1983) in: Ether Lipids: Biochemical and Biomedical Aspects (Mangold, H.K. and Paltauf, F. eds) pp.231-238, Academic Press, New York.

[6] Bandi, Z.L., Mangold, H.K., Holmer, G. and AaesJorgensen, E. (1971) FEBS Lett. 12, 217-220.

[7] Snyder, F. and Pfleger, R.C. (1966) Lipids 1, 328-334.

[8] Ahrne, L., Bjorck, L. and Claesson, O. (1982) Ann. Nutr. Metab. 26, 162170.

[9] Webber, K.O., Datta, N.S. and Hajra, A.K. (1987) Arch. Biochem. Biophys. 254, 611-620.

[10] Schutgens, R.B.H., Heymans, H.S.A., Wanders, R.J.A., Van den Bosch, H. and Tager, J.M. (1986) Eur. J. Ped. $144,430-444$.

[11] Heymans, H.S.A., Schutgens, R.B.H., Tan, R., Van den Bosch, H. and Borst, P. (1983) Nature 306, 69-70.

[12] Wilson, G.N., Holmes, R.G., Custer, J., Lipkowitz, J.L., Stover, J., Datta, N.S. and Hajra, A.K. (1986) Am. J. Med. Genet. 24, 69-82,

[13] Das, A.K. and Hajra, A.K. (1984) Biochim. Biophys. Acta 796, 178-189.

[14] Baumann, W.J. and Mangold, H.K, (1964) J. Org. Chem. 29, 3055-3057.

[15] Hajra, A.K. and Radin, N.S. (1963) J. Lipid Res. 4, $448-453$.

[16] Wells, M.A. and Dittmer, J.C. (1966) Biochemistry 5, 3405-3418.

[17] Hofle, G. and Steglich, W. (1972) Synthesis, pp.619-621.

[18] Horrocks, L.A. (1968) J. Lipid Res. 9, 469-472.

[19] Bartlett, G.R. (1959) J. Biol. Chem. 234, 466-468.

[20] Hajra, A.K., Saraswathi, T.V. and Das, A.K. (1983) Chem. Phys. Lipids 33, 179-193.

[21] Horrocks, L.A. (1972) in: Ether Lipids: Chemistry and Biology (Snyder, F. ed.) pp.177-272, Academic Press, New York.

[22] Chae, K., Piantadosi, C. and Snyder, F. (1973) J. Biol. Chem. 248, 6718-6723.

[23] Fleming, P.J. and Hajra, A.K. (1977) J. Biol. Chem. 252, $1663-1672$.

[24] Hajra, A.K. (1983) in: Ether Lipids (Mangold, H.K. and Paltauf, F. eds) pp.85-106, Academic Press, New York.

[25] Tietz, A., Lindberg, M. and Kennedy, E.P. (1964) J. Biol. Chem. 239, 4081-4090.

[26] Weber, N. (1985) J. Lipid Res. 26, 1412-1420.

[27] Bishop, J.E. and Hajra, A.K. (1981) J. Biol. Chem. 256, 9542-9550.

[28] Paltauf, F. (1983) in: Ether Lipids: Biochemical and Biomedical Aspects (Mangold, H.K. and Paltauf, F. eds) pp.107-128, Academic Press, New York.

[29] Reichwald, I. and Mangold, H.K. (1977) Nutr. Metab. suppl.21, 198-201.

[30] Moser, H.W. (1986) J. Pediatr. 108, 89-91. 